\title{
Characterizing Sub-lattice Occupancies in B2 Phases in High Entropy Metallic Alloys using Atomic Resolution STEM-XEDS Mapping
}

\author{
Robert E. A. Williams ${ }^{1}$, Brian Welk ${ }^{1}$, Bryan D. Esser ${ }^{1}$, Gopal B. Viswanathan ${ }^{1}$, Arda Genc ${ }^{2}$, Mark \\ Gibson $^{3}$, Leslie J. Allen ${ }^{4}$, David W. McComb ${ }^{1}$ and Hamish L. Fraser ${ }^{1}$ \\ ${ }^{1}$ Center for the Accelerated Maturation of Materials, Department of Materials Science and Engineering, \\ The Ohio State University, Columbus, OH 43212 \\ ${ }^{2}$ FEI Company, Hillsboro, OR \\ ${ }^{3}$ CSIRO, Clayton, Vic 3169, Australia \\ ${ }^{4}$ School of Physics, University of Melbourne, Parkville, Victoria 3010, Australia
}

In the recent past, there has been considerable emphasis placed on the exploration of high entropy alloys (HEA). These alloys have been defined as ones with five or more, essentially equal atomic concentrations $[1,2]$. CoCrCuFeNiAl is an example of an HEA alloy which mainly consists of two phases, namely ordered B2 and disordered bcc. Although this alloy has been the subject of much study and its microstructures characterized using a number of techniques, only recently has aberration-corrected (S)TEM coupled with x-ray energy dispersive spectroscopy (XEDS), involving large collection angles (ChemiSTEM ${ }^{\mathrm{TM}}$ ), been applied [3]. In this latter study, it was found that, between the $\mathrm{B} 2$, consisting mainly of $\mathrm{Al}$, and $\mathrm{Ni}, \mathrm{Co}$, and $\mathrm{Fe}$, and disordered $b c c$ phases, consisting mainly of $\mathrm{Cr}$ and $\mathrm{Fe}$, there is a transition region, approximately $1.5 \mathrm{~nm}$ in width, over which the chemical composition changes from the B2 to that of the $b c c$ phase. The crystal structure of this interfacial region is also B2, but with a significantly different sub-lattice occupancy than that of the adjacent B2 compound. In these B2 phases with very differing compositions, and hence sub-lattice compositions, the intensities of both atomic columns in HAADF images and superlattice reflections in diffraction patterns may vary considerably [3]. The origin of these intensity differences is of interest. For example, when the difference in the intensities of atomic columns in each of the sub-lattices is small, this may be interpreted as either the average compositions of the sub-lattices being similar, and/or being a reduced degree of order of the B2 compound. It is obvious that in order to be able to understand the behavior of these alloys, it is necessary that the degree of order be known. The first of these possibilities may be assessed by making direct measurements of the sub-lattice composition, while the second possibility, degree of ordering, may be assessed by plotting these compositions on an ordering tie-line diagram [4]. The current study involves the direct measurement of sub-lattice compositions.

Figure 1 shows the collection of spatially resolved XEDS maps from a sample of $\mathrm{CoCrCuFeNiAl}_{1.5}$, which may be used to yield the first approximation of the compositions on the two sub-lattices of the B2 compound. We believe that this is the first such atomically-resolved XEDS measurement on a metallic alloy. To further refine the compositional data so that accurate compositional information may be determined, it is necessary to consider the origin of the various signals that contribute to the recorded spectra. In recent work, the influence of phonon scattering to spatially-resolved XEDS and EELS measurements has been assessed [5,6]. For refinement of the present XEDS data, it is necessary to account for any spectral contributions from such inelastic scattering, and this is the subject of current research. 


\section{References:}

[1] J.W. Yeh, et al, Advanced Engineering Materials 6, (2004)

[2] C. J. Tong, et al, Metallurgical and Materials Transactions A, 36, (2005)

[3] B. Welk, et al, Ultramicroscopy 134, (2014)

[4] D.H. Hou, I.P. Jones and H.L.Fraser, Phil. Mag., A74, 741 (1996)

[5] A.D. d'Alfonso, Physical Review B 81, (2010)

[6] BD Forbes, et al, Phys. Rev. B. 82 (2010)

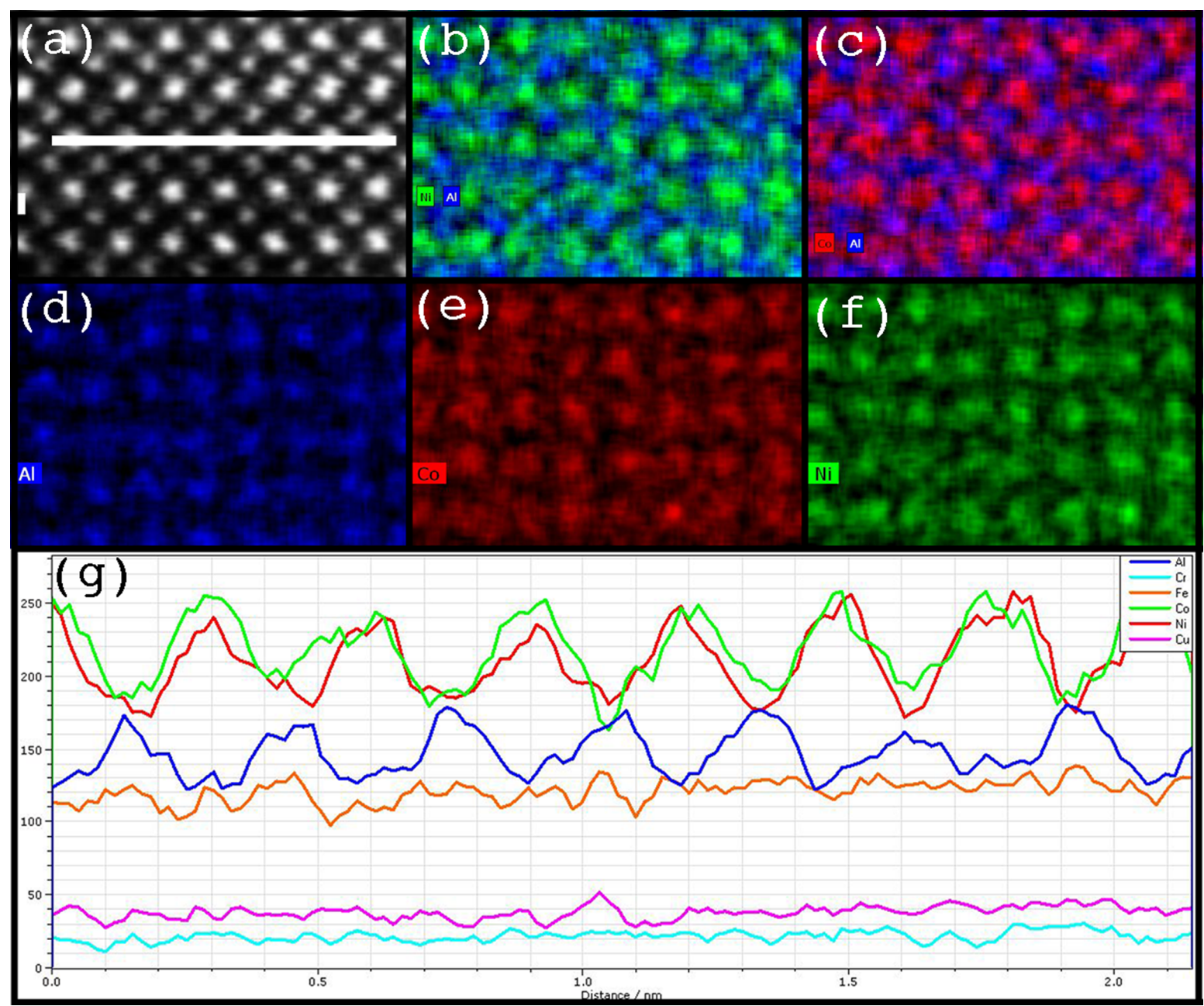

Figure 1: (a) STEM-HAADF image showing B2 ordering and line profile for (g). (b) Ni/Al EDS map, (c) Co/Al EDS map, (d) Al EDS map, e) Co EDS map, (f) Ni EDS map, (g) integrated line profile showing apparent atomic level chemical modulations with B2 lattice. 